\title{
One-electron atoms in Schwarzschild universe: bare and electromagnetically dressed cases
}

\author{
Abolfazl Jafari ${ }^{\mathrm{a}}$ \\ Department of Physics, Faculty of Science, Shahrekord University, P. O. Box 115, Shahrekord, Iran
}

Received: 13 February 2014 / Accepted: 27 May 2014 / Published online: 24 June 2014

(c) The Author(s) 2014. This article is published with open access at Springerlink.com

\begin{abstract}
The quantum mechanics of one-electron atoms in the presence of external electromagnetic fields is considered within Weber's framework. The results by the earlier studies are extended in the sense that for given source and field configurations the changes of the electromagnetic potentials due to the curved background are included. The formulation is specialized to the case with Schwarzschild background. The first corrections to the energy levels for bare atom and Zeeman/Stark effects are calculated, exhibiting possible changes in meaningful orders.
\end{abstract}

\section{Introduction}

The behavior of quantum mechanical systems in the presence of gravitational fields has been the subject of a great number of research publications. Among others, two leading approaches are those by DeWitt [1] and Weber [2]. In the studies based on DeWitt's approach, the general formulation of quantum mechanics for a relativistic or nonrelativistic system on a curved background is the main concern [3-6]. In the latter, based on Weber's, an interaction scheme between the quantum system and the gravitational field is the guiding rule. In particular, in this approach the linearized classical equations of motion of the test system/particle interacting with the gravitational fields provide the basic ingredients to formulate the quantum theory [2,7,8]. Interestingly, these two approaches are not equivalent, and they are based on different sequences and orders of approximations being used in each approach; one may get different results $[7,8]$.

Based on the DeWitt approach, the formulation of Dirac particles on a curved background is used to extract the first corrections in curvature to the energy levels of one-electron atoms [3-6]. In [7,8], the interaction between gravitational waves and charged test particle is studied. While [7,8] falls within Weber's scheme, it is shown that the sequence of lin-

\footnotetext{
a e-mail: jafari-ab@sci.sku.ac.ir
}

earizations used in the original version [2] is not sufficient in the case that one is dealing with the charged particles in the presence of external fields.

The purpose of the present work is to extend the results for nonrelativistic charged particles on a curved background. In particular, within Weber's framework, we consider the case with one-electron atoms in the presence of additional external electromagnetic fields in the small curvature limit to obtain the first corrections to the energy levels. Extending the results by [3-5], for given source or field configurations, the corrections due to curvature to the electromagnetic potentials as well as and their effects on the energy levels are studied. It will be seen that the obtained corrections to the nuclei potential and the external fields due to curvature can result in changes in the meaningful orders of magnitude. As a specific example, the corrections to the energy levels of the one-electron atom in the Schwarzschild metric is considered.

The scheme of the rest of this paper is the following. In Sect. 2, the basic notions of the formulation on curved background, including the Riemann normal coordinate system is reviewed. In Sect. 3, the basic elements of the quantization procedure as well as the construction of the Hamiltonian in the presence of the electromagnetic potentials based on Weber's approach are presented. In Sect. 4 the formulation is specialized to the case of a one-electron atom in a Schwarzschild background. In particular, for the case of a bare atom and the Zeeman/Stark effects the first corrections to the energy levels are obtained. Section 5 is devoted to our concluding remarks.

\section{Basic notions}

According to general relativity principles, it is not possible to find a system of coordinates in curved space-time in which $\Gamma_{\alpha \beta}^{\gamma}=0$ everywhere $(\alpha, \beta, \gamma=0,1,2,3)$. However, one always can construct local inertial frames at a given event 
$P_{0}$, in which free particles would move along straight lines locally. As a consequence, it is possible to set $\Gamma_{\alpha \beta}^{\gamma}=0$ at least up to the first order of the Riemannian curvature. As the constructed tangent space is similar to the Minkowski spacetime, a local inertial frame is defined for each given point $P_{0}$ of space-time by the following equation for the metric:

$g_{\alpha \beta, \mu}\left(P_{0}\right)=0$.

The coordinates of such a frame are called the Riemann normal coordinate system [2,9-12]. The metric components have the following forms in the Riemann coordinates up to the first order of Riemann's tensor $(i, j, \cdots=1,2,3)$ :

$$
\begin{aligned}
& g_{00}=-1-R_{0 l 0 k} x^{l} x^{k}, g_{0 i}=g^{0 i}=-\frac{2}{3} R_{0 l i k} x^{l} x^{k}, \\
& g_{i j}=\delta_{i j}-\frac{1}{3} R_{i l j k} x^{l} x^{k}, g^{00}=-1+R_{0 l 0 k} x^{l} x^{k}, \\
& g^{i j}=\delta^{i j}+\frac{1}{3} R_{l k}^{i j} x^{l} x^{k}, g=-1+\frac{1}{3}\left(R_{l k}-2 R_{0 l 0 k}\right) x^{l} x^{k} .
\end{aligned}
$$

Consequently, the affine connections (Christoffel multipliers) are found to be

$$
\begin{aligned}
& \Gamma_{00}^{0}=0, \quad \Gamma_{i j}^{0}=\frac{1}{3}\left(R_{0 i j k}+R_{0 j i k}\right) x^{k}, \\
& \Gamma_{0 i}^{0}=R_{0 i 0 k} x^{k}, \quad \Gamma_{j k}^{i}=\frac{1}{3}\left(R_{j i k l}+R_{k i j l}\right) x^{l}, \\
& \Gamma_{0 j}^{i}=R_{0 k j i} x^{k}, \quad \Gamma_{00}^{i}=R_{0 i 0 k} x^{k} .
\end{aligned}
$$

The equation of the motion of a test particle in an arbitrary coordinate system reads

$m \frac{d^{2} x^{\mu}}{d \tau^{2}}+m \Gamma_{\alpha \beta}^{\mu} u^{\alpha} u^{\beta}=F^{\mu}+\frac{q}{c} F_{\mathrm{em}}^{\mu \alpha} u^{\beta} g_{\alpha \beta}$,

with $u^{\alpha}=d x^{\alpha} / d \tau$ the velocity four-vector and $\tau$ the proper time. $F_{\mathrm{em}}^{\mu \alpha}$ and $F^{\mu}$ stand for the space-time components of the electromagnetic and other external forces acting on the particle, respectively. The above equation of motion can be obtained in terms of the metric $g_{\alpha \beta}(x)$ and the electromagnetic potential four-vector $A^{\alpha}$, usually adopted by the Lorentz gauge, from the following Lagrangian $[9,11,12]$ :

$\mathcal{L}=\frac{1}{2} m \dot{x}^{\alpha} g_{\alpha \beta}(x) \dot{x}^{\nu}+\frac{q}{c} \dot{x}^{\alpha} g_{\alpha \beta}(x) A^{\beta}-\tilde{V}(x)$,

in which

$$
-\int^{x} F_{\mu} d x^{\mu}=\tilde{V}(x)
$$

\section{Toward quantum system}

Here, using a set of assumptions and approximations, we develop the quantum mechanics governing the dynamics of the test particle. As announced earlier, our approach is basically the one by Weber's.

As we are considering nonrelativistic dynamics, it is assumed that the proper time can simply be replaced by the coordinate time $x^{0}$, by $\tau \rightarrow c x^{0}$, by which the equations of motion of the test particle for spatial directions become

$$
\begin{aligned}
& m \ddot{x}_{i}+m R_{0 i 0 j} x^{j}+\frac{1}{3} m\left(R_{j i k l}+R_{k i j l}\right) x^{l} \dot{x}^{j} \dot{x}^{k} \\
& =F^{i}+\Sigma_{j} \frac{q}{c} F_{i j}^{\mathrm{em}} \dot{x}_{j}+\frac{q}{c} F_{i 0}^{\mathrm{em}} .
\end{aligned}
$$

Now and hereafter, we consider the cases for which we have

$g_{0 i}=0$.

Many interesting cases, including the Schwarzschild metric, are of this type. Thus we introduce the following Lagrangian:

$$
\begin{aligned}
\mathcal{L}= & \frac{1}{2} m \dot{x}^{i} \delta_{i j} \dot{x}^{j}-\frac{1}{6} m R_{i s j k} \dot{x}^{i} x^{s} x^{k} \dot{x}^{j}+\frac{q}{c} \dot{x}^{i} \delta_{i j} A^{j} \\
& -\frac{q}{3 c} R_{i s j k} \dot{x}^{i} x^{s} x^{k} A^{j}-\tilde{V}_{\mathrm{eff}}(x),
\end{aligned}
$$

where

$\tilde{V}_{\mathrm{eff}}(x)=\tilde{V}(x)-\frac{1}{2} m c^{2} g^{00}-\frac{q}{c} A_{0}$.

The following is to show that the above Lagrangian produces the desired equations of motion of the test particle according the Weber's picture. Firstly, it is pointed out that the raising of the Lorentz indices is done with the metric (2), namely, $A_{i}=g_{i j} A^{j} \sim\left(\delta_{i j}-\frac{1}{3} R_{i l j k} x^{l} x^{k}\right) A^{j}$. Further, in the weak-field limit, the gravitational force is given by $F_{\text {grav }}^{\alpha}=-\frac{1}{2} m c^{2} \frac{\partial}{\partial x_{\alpha}} g^{00}[11,12]$, so we need to keep the velocity-independent term appearing in Eq. (5). Therefore, the gravitational parts will not be included in $\tilde{V}_{\text {eff }}(x)$. We mention that the metric is not explicitly a function of time. For the sake of simplicity, we set $m=1, \frac{q}{c} A_{\mu} \rightarrow A_{\mu}$ :

$$
\begin{aligned}
\frac{d}{d t} \frac{\partial \mathcal{L}}{\partial \dot{x}_{k}}-\frac{\partial \mathcal{L}}{\partial x_{k}}= & g^{k i} \ddot{x}_{i}+\dot{g}^{k i} \dot{x}_{i}+\dot{g}^{k i} A_{i}+g^{k i} \dot{A}_{i} \\
& -\frac{1}{2} \dot{x}_{i} \dot{x}_{j} g^{i j, k}-g^{i j, k} \dot{x}_{i} A_{j}-\dot{x}_{i} g^{i j} A_{j}^{, k} \\
& +\tilde{V}^{, k}-\frac{1}{2} g^{00, k}-A_{0}^{, k}=0 .
\end{aligned}
$$

Using $\dot{g}^{k i} \dot{x}_{i}-\frac{1}{2} \dot{x}_{i} \dot{x}_{j} g^{i j, k}=\Gamma_{i j}^{k} \dot{x}^{j} \dot{x}^{i}$ and $\Gamma_{00}^{k}=-\frac{1}{2} g_{00}^{, k}$, and referring to (8), $\Gamma_{0 j}^{k}=0, A_{0}^{, k}=F^{0 k} g_{00}$, and 


$$
\begin{aligned}
& \dot{g}^{k i} A_{i}+g^{k i} \dot{A}_{i}-g^{i j, k} \dot{x}_{i} A_{j}-\dot{x}_{i} g^{i j} A_{j}^{, k} \\
& =g^{k j, i} \dot{x}_{i} A_{j}+g^{k j} \dot{x}_{i} A_{j}^{, i}-g^{i j, k} \dot{x}_{i} A_{j} \\
& \quad-\dot{x}_{i} g^{i j} A_{j}^{, k}=\partial^{i}\left(g^{k j} A_{j}\right) \dot{x}_{i}-\partial^{k}\left(g^{i j} A_{j}\right) \dot{x}_{i} \\
& =A^{k, i} \dot{x}_{i}-A^{i, k} \dot{x}_{i}=F^{k i} \dot{x}^{j} g_{j i} .
\end{aligned}
$$

It is seen that the Lagrangian (9) can produce the equation of the motion (7). The Hamiltonian of the system can be constructed easily using the Legendre transformation. The conjugate momentum is as follows:

$p_{k}=m \dot{x}^{i} \delta_{i k}-\frac{1}{3} m R_{k j i l} x^{j} x^{l} \dot{x}^{i}+\frac{q}{c} A^{j} \delta_{j k}-\frac{q}{3 c} R_{k j i l} x^{j} x^{l} A^{i}$,

by which

$m \dot{x}^{i}\left(\delta_{i k}-\frac{1}{3} R_{k j i l} x^{j} x^{l}\right)=p_{k}-\frac{q}{c} \delta_{k i} A^{i}+\frac{q}{3 c} R_{k j i l} x^{j} x^{l} A^{i}$.

By the set of coordinates and their conjugate momenta, the Hamiltonian gets the form

$$
\begin{aligned}
\mathcal{H}= & \dot{x}^{i} p_{i}-\mathcal{L} \\
= & \frac{1}{2} m \dot{x}^{i} \delta_{i j} \dot{x}^{j}-\frac{1}{6} m R_{i k j l} \dot{x}^{i} x^{k} x^{l} \dot{x}^{j}+\tilde{V}_{\mathrm{eff}} \\
= & \frac{1}{2} \dot{x}^{i}\left(m \dot{x}^{j}\left(\delta_{i j}-\frac{1}{3} R_{i k j l} x^{k} x^{l}\right)\right)+\tilde{V}_{\mathrm{eff}} \\
= & \frac{1}{2}\left(\dot{x}_{s}\left(\delta^{s i}+\frac{1}{3} R_{q r}^{s i} x^{q} x^{r}\right)\right)\left(m \dot{x}^{j}\left(\delta_{i j}-\frac{1}{3} R_{i k j l} x^{k} x^{l}\right)\right)+\tilde{V}_{\mathrm{eff}} \\
= & \frac{1}{2 m}\left(p^{k}-\frac{q}{c} \delta^{i k} A_{i}-\frac{q}{3 c} R_{j n}^{k i} x^{j} x^{n} A_{i}\right) \\
& \times\left(p_{k}-\frac{q}{c} \delta_{k s} A^{s}+\frac{q}{3 c} R_{k l s r} x^{l} x^{r} A^{s}\right)+\tilde{V}_{\mathrm{eff}} \\
= & \frac{1}{2 m} p_{k} p^{k}-\frac{q}{2 m c} p^{k} \delta_{k i} A^{i}-\frac{q}{2 m c} A_{i} \delta^{i k} p_{k} \\
& +\frac{q}{6 m c} R_{k j i l} p^{k} x^{j} x^{l} A^{i}-\frac{q}{6 m c} R_{j l}^{k i} x^{j} x^{l} A_{i} p_{k} \\
& +\frac{q^{2}}{2 m c^{2}} A_{i} \delta^{i k} \delta_{k i} A^{i}-\frac{q^{2}}{6 m c^{2}} R_{k j i l} \delta^{i k} A_{i} x^{j} x^{l} A^{i} \\
& +\frac{q^{2}}{6 m c^{2}} R_{j l}^{k i} x^{j} x^{l} A_{i} \delta_{k i} A^{i}+\tilde{V}_{\mathrm{eff}},
\end{aligned}
$$

or, equivalently, it can be reduced to the following:

$$
\begin{aligned}
\mathcal{H}= & \frac{1}{2 m} \mathbf{p}^{2}-\frac{q}{2 m c}\left(p^{k} \delta_{k i} A^{i}+A_{i} \delta^{i k} p_{k}\right) \\
& +\frac{q}{6 m c}\left(R_{k j i l} p^{k} x^{j} x^{l} A^{i}-R_{j l}^{k i} x^{j} x^{l} A_{i} p_{k}\right) \\
& +\tilde{V}_{\mathrm{eff}}+O(\mathbf{A})^{2},
\end{aligned}
$$

where the Coulomb potential appearing in $\tilde{V}_{\text {eff }}(x)$ is given by the Maxwell equations in the curved background.

Here we assume that the solutions to the Maxwell equations for the potentials $A_{i}$ are subjected to the Coulomb gauge, by which $\hat{p}^{k} \delta_{k i} \hat{A}^{i}=\hat{A}_{i} \delta^{i k} \hat{p}_{k}$,

where the symbol ${ }^{\wedge}$ indicates that the operator forms of the variables are being used. It is easy to check that due to the diagonal form of Riemann's curvature tensors of the Schwarzschild universe, the above form is possible.

In passing to quantum theory, the classical values are replaced by their operator counterparts. Due to terms involving coordinates and momenta, one encounters the known problem of the ordering ambiguity. Here we exploit the rising of the Latin indices to construct the symmetrical Weyl ordering, by which the Hamiltonian (15) gets the form

$$
\begin{aligned}
\hat{\mathcal{H}}= & \frac{1}{2 m} \hat{\mathbf{p}}^{2}-\frac{q}{m c} \hat{\mathbf{p}} \cdot \hat{\mathbf{A}}+\frac{q^{2}}{2 m c^{2}} \hat{\mathbf{A}} \cdot \hat{\mathbf{A}} \\
& +\frac{q}{3 m c} R_{i j i j} \hat{p}^{i} \hat{x}^{j} \hat{x}^{j} \hat{A}^{i} \\
& +\frac{q}{9 m c} R_{i j j i}\left(3 \hat{p}^{i} \hat{x}^{j} \hat{x}^{i} \hat{A}^{j}+2 \imath \hbar A^{j} \delta^{i i}+\imath \hbar x^{i} \partial^{i} A^{j}\right) \\
& +\hat{\tilde{V}}_{\mathrm{eff}}(\hat{x})+O(\mathbf{A})^{2} .
\end{aligned}
$$

In order to consider all of the corrections in first order of curvature, the electromagnetic potentials should also be recalculated. The Maxwell equations in the curved background take the following form:

$g^{\alpha \beta} \nabla_{\alpha} \nabla_{\beta} A_{\mu}-R_{\mu}^{v} A_{\nu}=-4 \pi J_{\mu}$,

for which, by applying the local coordinates introduced earlier, we have $[3,4]$

$$
\begin{aligned}
& \delta^{i j} \partial_{i} \partial_{j} A_{0}+\frac{1}{3} R_{i l j k} x^{k} x^{l} \partial^{i} \partial^{j} A_{0}+\frac{5}{3} R_{i 00 j} x^{j} \partial^{i} A_{0} \\
& +2 R_{i 0 j}^{k} x^{j} \partial^{i} A_{k}-\frac{2}{3} R_{j}^{i} x^{j} \partial_{i} A_{0}=-4 \pi J_{0} \\
& \delta^{i j} \partial_{i} \partial_{j} A_{m}+\frac{1}{3} R_{i l j k} x^{k} x^{l} \partial^{i} \partial^{j} A_{m}-\frac{2}{3} R_{m}^{\mu} A_{\mu}-\frac{1}{3} R_{00 m}^{l} A_{l} \\
& +\frac{2}{3} \delta^{i j}\left(R_{m i k}^{\alpha}+R_{i m k}^{\alpha}\right) x^{k} \partial_{j} A_{\alpha}-\frac{1}{3} R_{00 j}^{i} x^{j} \partial_{i} A_{m} \\
& \quad-\frac{2}{3} R_{j}^{i} x^{j} \partial_{i} A_{m}=-4 \pi J_{m} .
\end{aligned}
$$

Using the perturbative expansion $A_{\alpha}=A_{\alpha}^{0}+A_{\alpha}^{1}+O(R)^{2}$, for the source of the nucleus $J_{0}=-Q \delta(\mathbf{r})$ and $J_{i}=0$, one finds the following for the electromagnetic potentials:

$$
\begin{aligned}
A_{0}^{\text {nucl. }}= & -Q r^{-1}+\frac{1}{12} Q\left(R+4 R_{00}\right) r \\
& +\frac{1}{12} Q\left(3 R_{j 0 k}^{0}-R_{j k}\right) x^{j} x^{k} r^{-1}, \\
A_{m}^{\text {nucl. }}= & \frac{1}{2} Q R_{0 m} r+\frac{1}{6} Q R_{j m k}^{0} x^{j} x^{k} r^{-1} .
\end{aligned}
$$

Assuming that the electromagnetic potentials have two parts, corresponding to the one by the nucleus of the one-electron 
atom and to the one by the external sources (as in Zeeman and Stark effects), we use the following replacement:

$A_{\alpha} \rightarrow A_{\alpha}^{\text {nucl. }}+A_{\alpha}$,

in which the second term is responsible for the potential by the external sources. The latter Hamiltonian takes the form

$$
\begin{aligned}
\hat{\mathcal{H}} \simeq & \frac{1}{2 m} \hat{\mathbf{p}}^{2}-Q e \hat{r}^{-1}+\frac{1}{12} Q e\left(\left(R+4 R_{00}\right) \hat{r}\right. \\
& \left.+\left(3 R_{j 0 k}^{0}-R_{j k}\right) \hat{x}^{j} \hat{x}^{k}\right) \hat{r}^{-1} \\
& +\frac{1}{2} m R_{0 s 0 k} \hat{x}^{s} \hat{x}^{k}+\frac{e^{2}}{2 m c^{2}} \hat{\mathbf{A}} \cdot \hat{\mathbf{A}}-\frac{e}{m c} \hat{\mathbf{p}} \cdot \hat{\mathbf{A}} \\
& +\frac{e}{3 m c} R_{i j i j} \hat{p}^{i} \hat{x}^{j} \hat{x}^{j} \hat{A}^{i} \\
& +\frac{e}{9 m c} R_{i j j i}\left(3 \hat{p}^{i} \hat{x}^{j} \hat{x}^{i} \hat{A}^{j}+2 \imath \hbar A^{j} \delta^{i i}+\imath \hbar x^{i} \partial^{i} A^{j}\right) .
\end{aligned}
$$

The above Hamiltonian is written in terms of local coordinates and is valid for the non-covariant observer as well. As a consequence, the results from the theory only can be interpreted in a local framework based on Riemann normal coordinates. As is evident, these two are identical in a Schwarzschild background without external electromagnetic fields.

\section{Quantum theory in Schwarzschild background}

As an application of the quantum theory developed in the previous section, here we consider the background by the static Schwarzschild solution

$$
\begin{aligned}
d s^{2}= & -c^{2}\left(1-\frac{2 G M}{c^{2} r}\right) d t^{2}+\left(1-\frac{2 G M}{c^{2} r}\right)^{-1} d r^{2} \\
& +r^{2}\left(d \theta^{2}+\sin ^{2} \theta d \phi^{2}\right),
\end{aligned}
$$

in which $r, \theta$, and $\phi$ represent the spherical coordinates. The nonvanishing $R_{\mu \nu \alpha \beta}$ are the relevant spatial components of the curvature tensor in the spherical coordinates given by

$$
\begin{aligned}
& R_{010}^{1}=-2 R_{020}^{2}=-2 R_{030}^{3}=\frac{2 G M\left(2 G M-r c^{2}\right)}{c^{4} r^{4}}, \\
& R_{232}^{3}=\frac{2 G M}{c^{2} r}, R_{131}^{3}=R_{121}^{2}=\frac{G M}{r^{2}\left(2 G M-r c^{2}\right)} .
\end{aligned}
$$

Evidently, the components of the curvature tensor would get arbitrary smaller values in the limit $r \rightarrow \infty$. This fact is the basis for using the results in the previous section which are valid in the small curvature limit. Therefore, for one-electron atoms sufficiently far from the origin, the quantum theory dev eloped in Sect. 3 is applicable.
4.1 Bare one-electron atom

Setting $A_{\alpha}^{0}=A_{a}^{1}=0$, we have

$\mathcal{H}=\hat{\mathcal{H}}_{0}+\frac{1}{2} m R_{0 i 0 j} \hat{x}^{i} \hat{x}^{j}+\frac{1}{4} Q e R_{i 0 j}^{0} \hat{x}^{i} \hat{x}^{j} r^{-1}$,

where $\hat{\mathcal{H}}_{0}$ stands for the unperturbed Hamiltonian of the oneelectron atom. The second term in the above represents the direct effect of the gravitational field on the energy, firstly considered by $[3,4]$ and used by [5] to obtain the corrections to the energy levels and the transition rates for large quantum numbers $n$. The third term in the above is evidently originating from the correction to the nuclei potential due to the curvature. As we will see, this added term would give comparable changes in the energy levels, specially for states with lower $n$. The typical radius of the curvature should be as small as $D \sim 10^{-3} \mathrm{~cm}$, by which in the nonrelativistic limit the perturbation would give larger corrections than the relativistic fine structure [3,4]. The following relations are useful in the subsequent calculations:

$$
\begin{aligned}
\frac{x^{2}}{r^{2}}= & \frac{\sqrt{4 \pi}}{3} Y_{0,0}(\theta, \phi)-\frac{1}{6} \sqrt{\frac{16 \pi}{5}} Y_{2,0}(\theta, \phi) \\
& +\frac{1}{4} \sqrt{\frac{32 \pi}{15}}\left(Y_{2,2}(\theta, \phi)+Y_{2,-2}(\theta, \phi)\right), \\
\frac{y^{2}}{r^{2}}= & \frac{\sqrt{4 \pi}}{3} Y_{0,0}(\theta, \phi)-\frac{1}{6} \sqrt{\frac{16 \pi}{5}} Y_{2,0}(\theta, \phi) \\
& -\frac{1}{4} \sqrt{\frac{32 \pi}{15}}\left(Y_{2,2}(\theta, \phi)+Y_{2,-2}(\theta, \phi)\right), \\
\frac{z^{2}}{r^{2}}= & \frac{\sqrt{4 \pi}}{3} Y_{0,0}(\theta, \phi)+\frac{1}{3} \sqrt{\frac{16 \pi}{5}} Y_{2,0}(\theta, \phi),
\end{aligned}
$$

and also

$$
\begin{aligned}
& \int_{0}^{\pi} d \theta \sin \theta \int_{0}^{2 \pi} d \phi Y_{l m}^{\star}(\theta, \phi) Y_{l_{1} m_{1}}(\theta, \phi) Y_{l_{2} m_{2}}(\theta, \phi) \\
& =\sqrt{\frac{\left(2 l_{1}+1\right)\left(2 l_{2}+1\right)}{4 \pi(2 l+1)}} C_{00,0}^{l_{1} l_{2}, l} C_{m_{1} m_{2}, m}^{l_{1} l_{2}, l},
\end{aligned}
$$

where $C_{m_{1} m_{2}, m}^{l_{1} l_{2}, l}$ stands for the Clebsch-Gordan coefficients. In what follows we represent the one-electron atom states as usual:

$|\psi\rangle=\left|n l m_{l}\right\rangle$,

where $n, l$, and $m_{l}$ are the relevant quantum numbers. Thus the first-order correction to the energy of the $S$-states $(l=0)$ is given by 
$\left\langle H_{S}^{1}\right\rangle=\frac{1}{4} Q e R_{i 0 j}^{0} \int d^{3} x \psi_{n 00}^{\star}(x) x^{i} x^{j} r^{-1} \psi_{n 00}(x)$.

Using the given form of $R_{0 i 0 j}$ by Eq. (27), we readily have

$R_{0101} x^{2}+R_{0202} y^{2}+R_{0303} z^{2}=R_{0303}\left(r^{2}-3 x^{2}\right)$,

by which for the $S$-states the first correction to the energy by the correction to the nuclei potential by the curvature vanishes. By this the present model coincides with those by Parker and Pinto's for $S$-states [3-5]. However, the situation is different for the $P$-states $(l=1)$. To calculate the $P$-states $(l=1)$ the diagonalization of the degenerate block of the Hamiltonian is required. Due to the electric quadrupole transition selection rules, we have $\Delta l=0, \pm 2$ and $\Delta m=0, \pm 1, \pm 2$. With a similar calculation for the $P$-states, one finds the matrix elements of the shifted Hamiltonian,

$$
\begin{gathered}
H_{m m^{\prime}}^{1}=\frac{1}{4} Q e R_{0303}\left(\langle\hat{r}\rangle_{n, 1}-3\left\langle\frac{\hat{x}^{2}}{\hat{r}}\right\rangle\right)=\frac{1}{4} Q e R_{0303}\langle r\rangle_{n, 1} \\
\times\left(C_{00,0}^{21,1} C_{0 m^{\prime}, m}^{21,1}-\sqrt{\frac{3}{2}} C_{00,0}^{21,1}\left(C_{2 m^{\prime}, m}^{21,1}+C_{-2 m^{\prime}, m}^{21,1}\right)\right),
\end{gathered}
$$

where $m$ and $m^{\prime}$ take the values $0, \pm 1$, corresponding to the $P_{x}, P_{y}$, and $P_{z}$ orbits, respectively. By setting $\beta=$ $\frac{1}{10} Q e R_{0303} \frac{\hbar^{2}}{m e^{2}}\left(3 n^{2}-2\right)$, and using the diagonal form of $R_{0 i 0 j}$, the explicit form of the matrix is found to be

$H_{m m^{\prime}}^{1}=\left(\begin{array}{ccc}-\frac{\beta}{4} & 0 & \frac{3 \beta}{4} \\ 0 & \frac{\beta}{2} & 0 \\ \frac{3 \beta}{4} & 0 & -\frac{\beta}{4}\end{array}\right)$.

By the corresponding eigenvalue equation,

$\operatorname{Det}\left(H_{m m^{\prime}}^{1}-E^{1} \delta_{m m^{\prime}}\right)=0$,

the following values are obtained for the corrections:

$E_{P}^{1}=\frac{\beta}{2},-\beta$.

As mentioned earlier, the correction to the nuclei potential is absent in [5]. As a consequence, in [5] the $S$-states, in agreement with the present model would not get corrections at first order. It would be useful to compare the result for the $P$-states. By the last expression in the above, we would get

$\frac{E_{\text {new }}^{1}}{E_{\text {Pinto }}^{1}} \sim 10^{-1} \frac{Q e\langle r\rangle}{m\left\langle r^{2}\right\rangle} \propto 10^{-1} \frac{e^{2}\left(3 n^{2}-l(l+1)\right)}{2 m a_{0} n^{3}(2 l+1)}$, in which $E_{\text {new }}^{1}$ and $E_{\text {Pinto }}^{1}$ are the first corrections due to the corrections to the nuclei potential by the present model, and the curvature by $[3,5]$. As is evident, the corrections by the additional term in (35) are not negligible for lower values of $n$ and have to be considered.

\subsection{The normal Zeeman effect}

Here we consider the effect of an external uniform magnetic field on the energy levels of the one-electron atom, which is, in the absence of spin effects, known as the normal Zeeman effect. For the nonzero uniform magnetic background field $\mathbf{B}=B_{0} \hat{k}$, due to the specific form of the Riemann tensor, it is easy to see that there is no change in the correction to the scalar potential $A_{0}$ of previous section, and we have

$A_{0}^{1}=\frac{1}{4} Q R_{j 0 k}^{0} x^{j} x^{k} r^{-1}$.

In the Coulomb gauge, the unperturbed components of the vector potential are given by

$A_{i}^{0}=-\frac{B_{0}}{2} \epsilon_{i j 3} x^{j}$.

The components of the correction to the potential, $A_{i}^{1}$, satisfy

$\partial^{2} A_{i}^{1}=\frac{B_{0}}{6}\left(3 R_{002}^{2}+2 R_{212}^{1}\right) \epsilon_{i j 3} x^{j}$,

by which, using $R_{0101}+R_{0202}+R_{0303}=0$, we have

$\mathbf{A}^{1}=\frac{B_{0}}{6}\left(3 R_{002}^{2}+2 R_{212}^{1}\right)\left(\frac{1}{3} y^{3}-y x^{2},-\frac{1}{3} x^{3}+x y^{2}, 0\right)$,

by which at the first order in curvature,

$\mathbf{A}=\left(\begin{array}{c}-\frac{B_{0}}{2} y+\frac{B_{0}}{6}\left(3 R_{002}^{2}+2 R_{212}^{1}\right)\left(\frac{1}{3} y^{3}-y x^{2}\right) \\ \frac{B_{0}}{2} x-\frac{B_{0}}{6}\left(3 R_{002}^{2}+2 R_{212}^{1}\right)\left(\frac{1}{3} x^{3}-x y^{2}\right) \\ 0\end{array}\right)$

By these the operator of the Dewitt Hamiltonian of the oneelectron atom for the normal Zeeman effect takes the form

$$
\begin{aligned}
\hat{\mathcal{H}}_{\text {Perturbed }}^{\text {Zeeman }} & -\frac{e B_{0}}{2 m c} \hat{\mathcal{L}}_{z}-\frac{e B_{0}}{6 m c}\left(3 R_{002}^{2}+2 R_{212}^{1}\right) \\
& \times\left(\hat{p}_{x}\left(\frac{1}{3} \hat{y}^{3}-\hat{y} \hat{x}^{2}\right)-\hat{p}_{y}\left(\frac{1}{3} \hat{x}^{3}-\hat{x} \hat{y}^{2}\right)\right) \\
& +O\left(B_{0}\right)^{2}
\end{aligned}
$$

in which the first two terms represent the unperturbed Hamiltonian. The above expression is valid for the so-called weak field, or $B_{0} \lesssim 10^{-2} \mathrm{G}$. In fact the last term above is the 
result of corrections to the potential. One can calculate the contribution of the correction related to the Zeeman effect, as follows:

$$
\begin{aligned}
\Delta E_{\text {Perturbed }}^{\text {Zeeman }}= & \Delta E_{0}^{\text {Zeeman }}+\left(\frac{e B_{0}}{18 m c}\left(3 R_{002}^{2}+2 R_{212}^{1}\right)\right. \\
& \times\left(n^{2} \frac{5 n^{2}+1-3 l(l+1)}{2} a_{0}^{2}\right) \\
& \left.\times m_{l} \hbar\left(1-C_{00,0}^{2 l, l} C_{0 m_{l}, m_{l}}^{2 l, l}\right)\right) .
\end{aligned}
$$

Evidently, there are nonzero corrections due to the second part for the $P$-states. The Weber Hamiltonian of the oneelectron atom for the normal Zeeman effect takes the form

$$
\begin{aligned}
\hat{\mathcal{H}}_{\text {Perturbed }}^{\text {Zeeman }} \simeq & -\frac{e B_{0}}{2 m c} \hat{\mathcal{L}}_{z}+\frac{e B_{0}}{6 m c} \epsilon^{j i} R_{i j i j} \hat{p}^{i} \hat{x}^{j} \hat{x}^{j} \hat{x}^{j} \\
& +\frac{e B_{0}}{18 m c} R_{i j j i} \epsilon^{i j} \\
& \times\left(3 \hat{p}^{i} \hat{x}^{j} \hat{x}^{i} \hat{x}^{j}+2 \imath \hbar \hat{x}^{i} \delta^{i i}+\imath \hbar \hat{x}^{i} \delta^{j j}\right) \\
& +O\left(B_{0}\right)^{2},
\end{aligned}
$$

in which one can transform to a more useful form by the identity

$\hat{p}_{x} \hat{y}^{3}-\hat{p}_{y} \hat{x}^{3}=\hat{\mathcal{L}}_{z}\left(\hat{r}^{2}-\hat{z}^{2}\right)+\hat{p}_{y} \hat{x} \hat{y}^{2}-\hat{p}_{x} \hat{y} \hat{x}^{2}$.

By the above, the contribution of the correction related to the Zeeman effect can be calculated. Following [5] and from a semi-classical point of view, we can calculate the energy levels based on the Bohr quantization procedure. In fact, the stability of motion of the one-electron atom in the $x y$ plane does not last long. However, after neglecting the effect of the last correction, the Bohr radius is definable. One can choose the orientation of the spatial axes of the normal coordinates such that $z \sim y^{\prime}, y \sim x^{\prime}$, and $x \sim z^{\prime}$. Now, if we restrict ourselves to circular orbits in the $x^{\prime} y^{\prime}$ plane and by assuming the presence of a magnetic field in the $z$ (radial) direction and with $\rho^{\prime}=\sqrt{x^{\prime 2}+y^{\prime 2}}$, the equation of motion can be shown to be

$m \frac{v^{\prime 2}}{\rho^{\prime}}=\frac{e v^{\prime} B_{0}}{c}+\frac{Q e}{\rho^{\prime 2}}+m R_{0202} \rho^{\prime}+\frac{Q e}{4} R_{0202}$

by the Bohr quantization condition $\left(m \rho v_{n}=n \hbar\right)$ and $R=$ $R_{0202}$, we get

$n^{2}-\frac{e B_{0}}{c \hbar} n \rho^{\prime 2}=\frac{z m e^{2}}{\hbar^{2}} \rho^{\prime}+\frac{m Q e R}{4 \hbar^{2}} \rho^{\prime 3}+\frac{m^{2} R}{\hbar^{2}} \rho^{\prime 4}$, or

$$
\begin{aligned}
\left(n-\frac{e B_{0}}{2 c \hbar} \rho^{\prime 2}\right)^{2}= & \frac{m Q e}{\hbar^{2}} \rho^{\prime}+\frac{m Q e R}{4 \hbar^{2}} \rho^{\prime 3} \\
& +\left(\frac{m^{2} R}{\hbar^{2}}+\frac{e^{2} B_{0}^{2}}{4 c^{2} \hbar^{2}}\right) \rho^{\prime 4}
\end{aligned}
$$

Comparing the above with a similar treatment by [5], the term proportional to $\rho^{\prime 3}$ is new. Therefore, although there is no change in the Landau-Bohr radius obtained in [5], however, a new radius can be defined by this new term, namely

$r_{a}=\left(\frac{4 \hbar^{2}}{3 m Q e R}\right)^{1 / 3}$

The obtained radius goes to infinity when $R=0$, leading to motion on a straight line.

\subsection{The Stark effect}

This energy shift of atomic levels in the presence of an external uniform electric field is known as the Stark effect. On this topic, the Hamiltonian describing the Stark effect is encountered with a technical agreement between the DeWitt and Weber approaches. The deformed Maxwell equations are used to reproduce the potential and redefine the distribution of electrical charges in order to produce a uniform electric fields in the presence of the gravitational background. To be specific and for the case of Weber's method, we assume that $J_{0}=Q \delta\left(\mathbf{r}_{-}\right)-Q \delta\left(\mathbf{r}_{+}\right)$, with $\mathbf{r}_{ \pm}=\mathbf{r} \pm \mathbf{R}$. Thus, if the size of $\mathbf{R}$ is infinite, then the electric field will be uniform. However, according to Eq. (20), the scalar potential gets the form

$A_{0}=A_{0}^{0}-\frac{1}{4} E_{0} R_{i 0 j}^{0} x^{i} x^{j} z$

where

$$
\begin{aligned}
A_{0}^{0} & =\frac{Q}{r_{-}}-\frac{Q}{r_{+}}=Q \Sigma_{0}^{\infty} \frac{r^{l}}{R^{l+1}}\left(P _ { l } \left(\cos (\gamma)-P_{l}(-\cos (\gamma))\right.\right. \\
& =\left.\frac{2 Q}{R^{2}} r \cos (\gamma)\right|_{\frac{2 Q}{R^{2}}=E_{0}},
\end{aligned}
$$

where $\gamma$ is the angle between $\mathbf{r}$ and $\mathbf{R}$. So, by setting $\mathbf{R}=R \hat{k}$, the potential by which the uniform electric field in curved background is produced is given by

$$
\begin{aligned}
A_{0} & =-E_{0} r \cos (\theta)-\frac{E_{0}}{4} R_{i 0 j}^{0} x^{i} x^{j} r \cos (\theta) \\
& =-E_{0} z-\frac{E_{0}}{4} R_{i 0 j}^{0} x^{i} x^{j} z .
\end{aligned}
$$


Hence, in Schwarzschild background, the perturbed Hamiltonian for the Stark effect is given by

$$
\begin{aligned}
& \hat{\mathcal{H}}_{\text {perturbed }}^{\text {Stark }} \simeq \mathcal{H}_{0}+\frac{1}{4} Q e R_{i 0 j}^{0} \hat{x}^{i} \hat{x}^{j} \hat{r}^{-1} \\
& \quad+\frac{1}{2} m R_{0 i 0 j} \hat{x}^{i} \hat{x}^{j}+e E_{0} \hat{z}+e \frac{E_{0}}{4} R_{i 0 j}^{0} \hat{x}^{i} \hat{x}^{j} \hat{z} .
\end{aligned}
$$

Due to the parity, the correction to the energy of the $S$-states by the term $e \frac{E_{0}}{4} R_{i 0 j}^{0} x^{i} x^{j} z$ vanishes.

\section{Concluding remarks}

The results for nonrelativistic charged particles on a curved background are extended. In particular, within the Weber framework, we consider the case with one-electron atoms in the presence of additional external electromagnetic fields in the small curvature limit to obtain the first corrections to the energy levels. Extending the results by [3-5], for given source or field configurations, the corrections due to curvature to the electromagnetic potentials as well as their effects on the energy levels are studied. It is seen that the obtained corrections to the nuclei potential and the external fields due to curvature can result in changes in meaningful orders of magnitude. As a specific example, the corrections to the energy levels of the one-electron atom in the Schwarzschild metric is considered. In particular, for the case with a bare atom it is observed for lower values of quantum number $n$ that the corrections to the scalar potential of the nucleus cannot be ignored and are comparable with the corrections by [5]. In the case of the Zeeman effect it is seen that, as is well known, the Hamiltonian would take a different form in comparison with that based on DeWitt's approach [5]. As a consequence, the semi-classical behavior of the systems would be different in comparison with a similar treatment of the system in [5].

Acknowledgments The author is grateful to A. H. Fatollahi for useful comments. Also the author thanks the Shahrekord University for support of this research grant fund.

Open Access This article is distributed under the terms of the Creative Commons Attribution License which permits any use, distribution, and reproduction in any medium, provided the original author(s) and the source are credited.

Funded by $\mathrm{SCOAP}^{3}$ / License Version CC BY 4.0.

\section{References}

1. B.S. DeWitt, Rev. Mod. Phys. 29, 377 (1957)

2. J. Weber., in General Relativity and Gravitational Waves, 1961, Dover Edition, 2004. Gravitational Radiation and Relativity, ed. by J. Weber, T.M. Karade, vol. 3 (Interscience Publisher Inc., New York) Proceedings of the Sir Arthur Eddington Centenary Symposium, Nagpur, India, 1984

3. L. Parker, Phys. Rev. Lett. 44, 1559 (1980)

4. L. Parker, Phys. Rev. D 22, 1922 (1980)

5. F. Pinto, Phys. Rev. Lett. 71, 1116 (1993)

6. L. Ramezan, M. Khorrami, Int. J. Theor. Phys. 49, 2918 (2010)

7. A.D. Speliotopoulos, Phys. Rev. D 51, 1701 (1995)

8. A. Saha., S. Gangopadhyay., S. Saha, Phys. Rev. D 83025004 (2011)

9. C.W. Misner, K.S. Thorne, J.A. Wheeler, Gravitation (Freeman Publishing Company, San Francisco 1973)

10. M. Maggiore, Gravitational Waves (Oxford University Press Inc., New York, 2008)

11. R. D'inverno, Introducing Einstein's Relativity (Oxford University Press Inc., New York, 1993)

12. S. Weinberg, Gravitation and Cosmology: Principles and Applications of the General Theory of Relativity (Wiley, New York, 1972) 